\title{
Representation of minority names on maps: the case of the Polish minority in the Těšín region, Czechia
}

\author{
Přemysl Mácha ${ }^{\mathrm{a}, *}$ \\ ${ }^{a}$ Institute of Ethnology, Czech Academy of Sciences, Brno, Czechia, macha@eu.cas.cz \\ * Corresponding author
}

Keywords: minority place names, maps, Poles, Czechia

\begin{abstract}
:
Representation of minority names on maps is a complex issue. It often generates political conflicts between the minority and the majority (and sometimes even other minorities). The inclusion of minority names on maps is an expression of a willingness to share the represented space with other groups. In this sense, place names function as symbolic claims, indicating the group's right to the territory. The majority may perceive it as a threat to their control of the land while the minority may see it as a recognition of their existence and right to home. Furthermore, the use of minority names on maps may also increase the relevance of the minority language and support the minority's linguistic vitality. Where more minorities are present, it becomes even more politically delicate to find generally accepted criteria for the inclusion of the names of a particular group (such as their share of the total population, territorial concentration, linguistic distinctiveness, etc.).
\end{abstract}

However, even in best-case scenarios, where there is good will on all sides, the inclusion of minority names on maps still presents a number of practical challenges with significant political and emotional consequences. A decision has to be made on the quantity, visual quality, and placement of the minority name label in relation to the already present labels. Which name should come first, the minority name or the majority? Should all labels look identically in terms of font, color, and size? How will the representation of minority names change with the scale of the map? Which minority names should be rendered on the map? Should only settlement names be represented or also names referring to natural features such as rivers, mountains, fields and forests? More importantly, a decision has to be made on the alphabet (in cases where the minority uses a different alphabet system) or the transcription of the name (in cases where the minority and the majority use the same alphabet but the names differ in form). And finally, it is often the case that the minority in reality speaks a specific local dialect in which the place name form differs from the form it has in the standard minority language. A choice between minority dialect and the minority standard language may also be a very salient issue potentially complicating negotiations about minority name representation on maps.

In my paper, I present the results of a study of minority name representation on maps of the Těšín region in eastern Czechia. It is a region with a traditional presence of Poles. Currently, Poles form up to $30 \%$ of local inhabitants in some communities. Historically, their share of the total population was significantly higher. It is also the only region in Czechia where a minority is sufficiently territorially concentrated to enjoy a right to bilingual signs in public spaces guaranteed by the Czech legislation. This encourages the minority to also demand representation of minority names on maps which is not guaranteed by law.

The introduction of bilingual signs as well as the inclusion of minority names on maps have been politically highly contentious issues in the past twenty years and the process is far from being resolved. The situation is further complicated by the strong presence of a local dialect used by the majority of Poles but also shared with the Czech majority. Toponymy is thus trilingual whereas official names only bilingual. This presents additional challenges for the decisions on which names in what form and transcription should be placed on maps of the region at which scale. In this paper, I first provide a historical overview of how minority names have been represented on maps of the region since the first detailed maps were published in 1700s to the current maps produced by the Czech state as well as local communities. Then, I give examples of several contentious maps which generated political conflicts at the local and regional levels. Finally, I generalize the findings in relation to previous studies and outline possibilities for further research. 